\title{
An Adaptation of the Wiener Filter Suitable for Analyzing Images of isolated Single Particles, and Accompanying 3D Reconstruction Algorithm
}

\author{
C.V. Sindelar* and N. Grigorieff** \\ * Department of Molecular Biophysics and Biochemistry, Yale University, New Haven, CT \\ 06520 \\ ** Howard Hughes Medical Institute, Department of Biochemistry, Rosenstiel Basic Medical \\ Sciences \\ Research Center, Brandeis University, 415 South Street, Waltham, MA 02454
}

The high noise level found in electron cryo-microscopy (cryo-EM) imaging data represents a special challenge for $2 \mathrm{D}$ and $3 \mathrm{D}$ reconstruction of the imaged molecules. The Wiener filter is a standard means of optimizing sums of aligned, noisy images, in order to obtain a least-squares estimate of the noise-free object being imaged [1,2]. A principle advantage of the Wiener filter is that if the "spectral" (i.e., resolution-dependent) signal-to-noise ratio (SSNR) for the raw data images is known or can be estimated as a function of resolution, this information can be used to reduce the error in the resulting estimate. However, while the Wiener filter has long been utilized in single-particle cryo-EM applications [3], estimation of the SSNR from the input data has remained problematic, and error reduction due to specific application of the SSNR term within a Wiener filter has not been reported. Instead, Wiener filters are typically applied with unrealistically high SSNR values in order to obtain reasonable particle estimates.

Here we show that a large adjustment to the Wiener filter is necessary, when applying the Wiener filter to images of isolated particles surrounded by large regions of featureless background, as is typically the case in single-particle cryo-EM applications. We derive an approximate expression for this adapted, "single-particle" Wiener filter, showing that the SSNR term found in the conventional Wiener filter must be scaled by a factor, $f$ particle, which reflects the fraction of the image field occupied by the particle. We also give related expressions that allow the SSNR to be computed with suitable accuracy for application in this new filter, by incorporating a masking step into a Fourier Ring Correlation (FRC), a standard resolution measure. Using numeric tests, we demonstrate that these new tools may be used to minimize the mean-squared error of 2D particle projection maps under realistic cryo-EM imaging conditions, yielding a "best estimate" of the particle given the available data.

We then derive and numerically validate a simple and efficient algorithm that extends this approach to three dimensions, approximating a least-squares solution for the 3D density of an imaged molecule from noisy image data when the viewing angles of the images are known. Using synthetically generated test data sets with realistic amounts of added noise, as well as experimentally generated data sets, we demonstrate that our new 3D algorithm reduces the mean squared error of single-particle reconstructions relative to currently available reconstruction methods as judged by various readouts of reconstruction quality, including the commonly used Fourier shell correlation metric (FSC). We also show that real-space maps produced by our algorithm have reduced mean-squared error relative to a noise-free reference map, when compared with a previously-proposed "figure-of-merit" reconstruction scheme [4]. In addition to providing improved reconstruction algorithms, the theoretical framework presented here clarifies the relationship between SNR and reconstruction quality for the case of isolated single particles. 


\section{References}

[1] N. Wiener, Extrapolation, interpolation, and smoothing of stationary time series, Technology Press MIT \& Wiley, 1949.

[2] A.N. Kolmogorov, Bull. Moscow Univ. 2 (no. 6) (1941) 1-40.

[3] W.O. Saxton, Computer Techniques for Image Processing in Electron Microscopy, Academic Press, New York, 1978.

[4] P.B. Rosenthal, R. Henderson, J. Mol. Biol. 333 (2003) 721-45.

[5] This research was supported by the Howard Hughes Medical Institute. 